\title{
Investigation of Trace Element Concentration in Diabetic Rat's Tissues
}

\author{
J. Kowalska ${ }^{a}$, M. Krośniak ${ }^{b}$, R. Gryboś ${ }^{c}$ And W.M. KwiateK ${ }^{a, *}$ \\ ${ }^{a}$ Department of Applied Spectroscopy, Institute of Nuclear Physics, PAS \\ Radzikowskiego 152, 31-342 Kraków, Poland \\ ${ }^{b}$ Department of Food Chemistry and Nutrition, Medical College Jagiellonian University \\ Kraków, Poland \\ ${ }^{c}$ Faculty of Chemistry, Jagiellonian University, Kraków, Poland
}

\begin{abstract}
Diabetes is one of the most frequent diseases in developing countries and thus there is a significant interest in diabetes related studies. It was found that vanadium compounds have glucose-lowering properties in diabetes and therefore it is very important to estimate the vanadium dose in diabetes treatment. On the other hand, the proper estimation of vanadium concentration is important due to side effects that occur in vanadium supplementation. In this study the influence of $\mathrm{V}(\mathrm{IV})$ and $\mathrm{V}(\mathrm{V})$ compounds with different ligands on the concentration of $\mathrm{K}$, Ca, Mn, $\mathrm{Fe}, \mathrm{Cu}$, and $\mathrm{Zn}$ in selected rat's tissues was investigated by means of proton induced X-ray emission technique. As a result of the measurements it was found that the concentration of vanadium depends on the organ. The highest value was determined in spleen while the lowest in pancreas. It was also found that the concentration of other elements depends on the presence of vanadium and its concentration. The most meaningful influence of vanadium presence was on iron concentration in spleen, on copper and zinc in kidney, and on manganese in pancreas.
\end{abstract}

PACS numbers: 29.30.Ep, 78.70.En, 87.19.X-

\section{Introduction}

Diabetes is one of the most frequent diseases in developing countries. Also, it has been estimated that over $1 \%$ of the human population in industrial countries is suffering from diabetes [1]. The prognosis for the next few years predicts a significant increase in the occurrence of this disease. There are two types of diabetes. The type I of diabetes is caused by the auto-immunological destruction of the $\beta$-cells in pancreas [2]. In this case the body does not produce insulin which is a hormone that is needed to convert sugar glucose into energy needed for daily life. In type II of diabetes either the body does not produce insulin or the tissues are insulin-resistant.

In rats' bodies the diabetes type I can be induced by streptozotocin. Some experiments performed in 80's of the XXth century have shown that vanadium compounds are characterized by properties which make the glucose level lower in diabetes [3, 4]. Since then, one is seeking for vanadium compounds which have a lowering influence on the glucose level without side effects such as vomiting, diarrhea, and lowering of body mass, which are observed after high dose of vanadium administration $[5,6]$. Therefore, the aim of this study was to investigate the influence of vanadium (IV) and (V) compounds on $\mathrm{K}, \mathrm{Ca}, \mathrm{Mn}, \mathrm{Fe}$, $\mathrm{Cu}$, and $\mathrm{Zn}$ concentrations in kidney, spleen, and pancreas.

* corresponding author; e-mail: Wojciech.Kwiatek@ifj.edu.pl

\section{Materials and method}

This research was performed on male Wistar rats in the age of $8-10$ weeks, in the weight of $180-240 \mathrm{~g}$. The animals were adapted for 12 hours day/night cycle, in room temperature with air humidity of $75-85 \%$. The rats were divided into 8 groups of 6 animals each. The first group was treated as control (C). After three days from the beginning of the experiment, the streptozotocin of $55 \mathrm{mg} / \mathrm{kg}$ body mass in citric buffer $(0.1 \mathrm{~mol} / \mathrm{l})$ was injected to rats from seven groups by caudal vein in volume of $1 \mathrm{ml} / \mathrm{kg}$ of body mass. The glucose level in blood measured after other three days was larger than $17 \mathrm{mmol} / \mathrm{l}$. Then animals were separated into tested groups: healthy control (C), diabetic control (D), diabetic with an injection of long-acting insulin (Di), and 5 diabetic groups with insulin injection and supplement with one of 5 vanadium complexes (Di1-Di5). The tested vanadium complexes (Fig. 1) in water solution were administered once a day by gavage in a dose of $50 \mu \mathrm{mol} / \mathrm{kg}$ body mass and additionally $1 \mathrm{U} / \mathrm{kg}$ body mass insulin was injected subcetenal. After five weeks the rats were anaesthetized by tiopentanal in a dose of $50 \mathrm{mg} / \mathrm{kg}$ body mass. Organs such as spleen, kidney, and pancreas were collected, frozen at $-20^{\circ} \mathrm{C}$, and kept frozen until performing the analysis. Table I shows the formula and structure of the tested vanadium complexes [7].

\subsection{Target preparation}

The frozen tissues were lyophilized in ABCONO FREEZONE 4.5 lyophilizing cabinet under pressure 


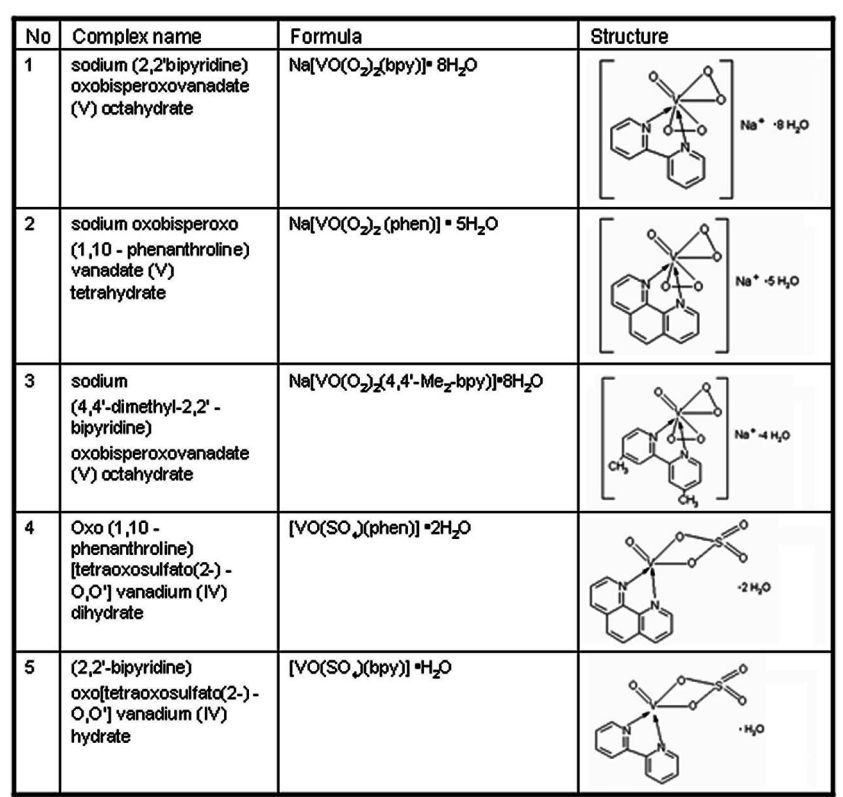

Fig. 1. Vanadium complexes used in this research.

ranging from $14 \mathrm{~Pa}$ to $1 \mathrm{~Pa}$ at temperature range form $-40^{\circ} \mathrm{C}$ to $-50^{\circ} \mathrm{C}$. Such prepared organs were powdered in the agate mortar in order to get a homogeneous material. The known amount of powder (about $80 \mathrm{mg}$ ) was pressed under pressure of $15 \mathrm{MPa}$ into pellets of about $10 \mathrm{~mm}$ in diameter and $1 \mathrm{~mm}$ thick. Each pellet was attached to the aluminum frame by a Scotch tape [8].

\subsection{PIXE analysis}

The proton induced X-ray emission (PIXE) method was applied in this study to investigate the concentration of $\mathrm{K}, \mathrm{Ca}, \mathrm{Mn}, \mathrm{Fe}, \mathrm{Cu}$, and $\mathrm{Zn}$. The experiment was performed at the Institute of Nuclear Physics, Polish Academy of Sciences in Kraków. A proton beam of 2.2 MeV from the Van de Graaff accelerator was used. The acquisition time of characteristic X-ray spectrum was set to $1200 \mathrm{~s}$ for each sample which corresponded to the charge of about $0.5 \mu \mathrm{C}$. The induced X-rays were registered by the CANBERRA $\mathrm{Si}(\mathrm{Li})$ detector with a resolution of $160 \mathrm{eV}$ for the energy of $5.9 \mathrm{keV}$. The backscattered protons were registered by a silicon surface-active detector and used for X-ray spectra normalization $[9,10]$. For quantitative determination of trace elements concentration external standards were used: IAEA H-8 Horse Kidney and National Standards \& Technology Standard Reference Material 1577b Bovine Liver [11].

\section{Results and discussion}

All results were calculated using the well-established WinGupix software package version 2.0 [12], while statistics calculation was done with Statistica version 7.0. The Turkey test $(p<0.05)$ was applied [13]. All results for each element are shown in Figs. 2-8, while Fig. 9 gives the mean values of these concentrations. Figures 10, 11, and 12 show significant statistical differences between tested groups.

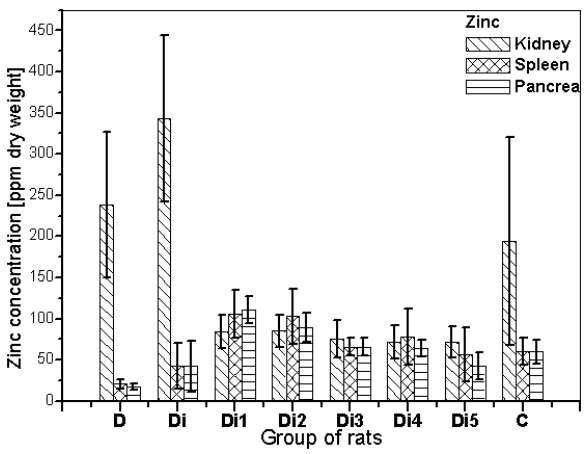

Fig. 2. Zinc concentration in organs.

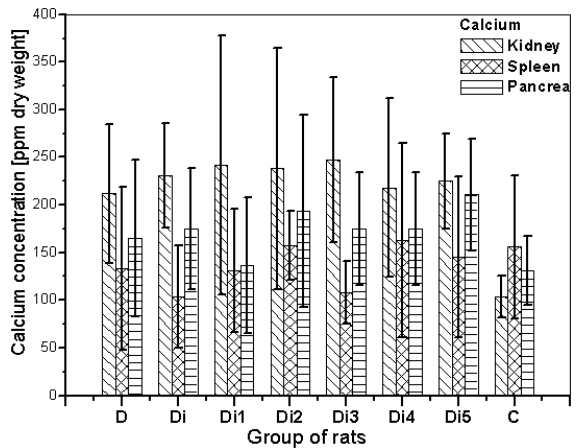

Fig. 3. Manganese concentration in organs.

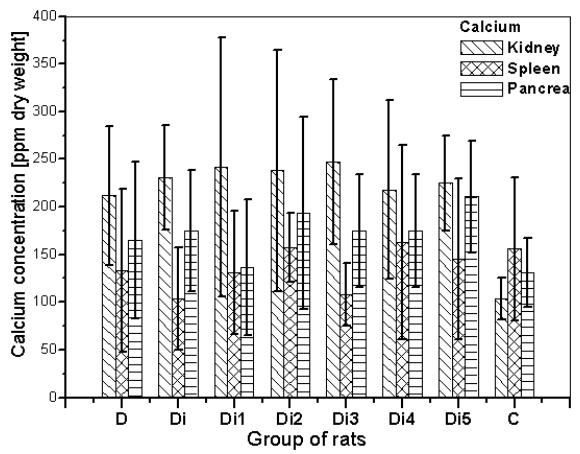

Fig. 4. Calcium concentration in organs.

It was found that vanadium supplementation has an influence on $\mathrm{Zn}, \mathrm{Mn}, \mathrm{Ca}, \mathrm{Cu}, \mathrm{Fe}, \mathrm{K}$, and $\mathrm{V}$ concentration in kidneys, on $\mathrm{Zn}, \mathrm{Mn}, \mathrm{Cu}, \mathrm{Fe}, \mathrm{K}$, and $\mathrm{V}$ in spleens and on $\mathrm{Zn}, \mathrm{Mn}, \mathrm{Fe}, \mathrm{K}$, and $\mathrm{V}$ in pancreas. Also diabetes influence was observed on $\mathrm{Cu}$ concentration in kidney, $\mathrm{Zn}$ and $\mathrm{Cu}$ concentration in spleens, and $\mathrm{Zn}$ and $\mathrm{Fe}$ concentration in pancreas. 


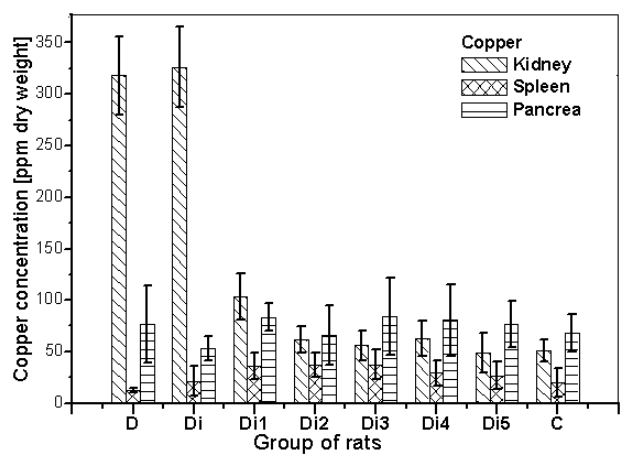

Fig. 5. Copper concentration in organs.

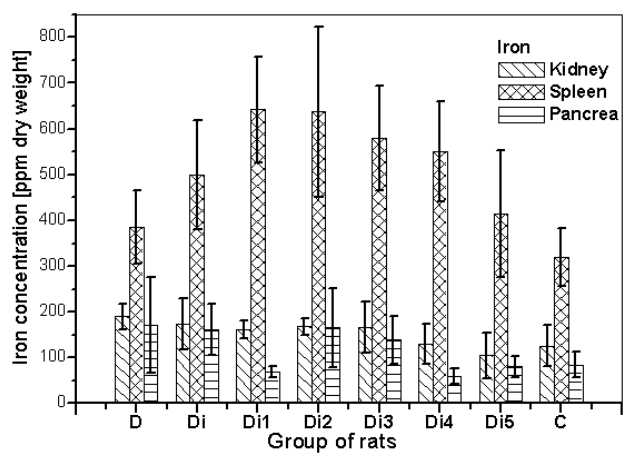

Fig. 6. Iron concentration in organs.

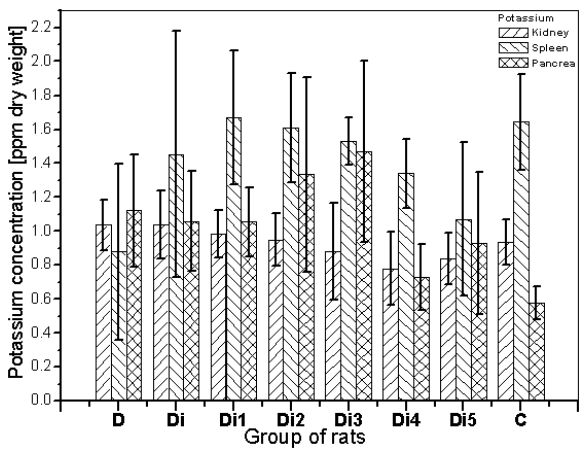

Fig. 7. Potassium concentration in organs.

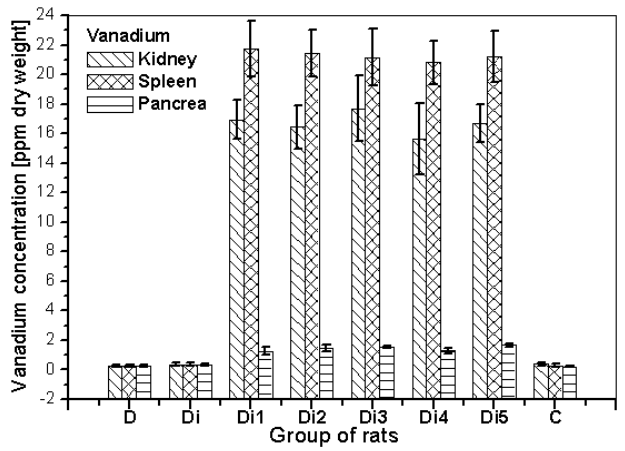

Fig. 8. Vanadium concentration in organs.

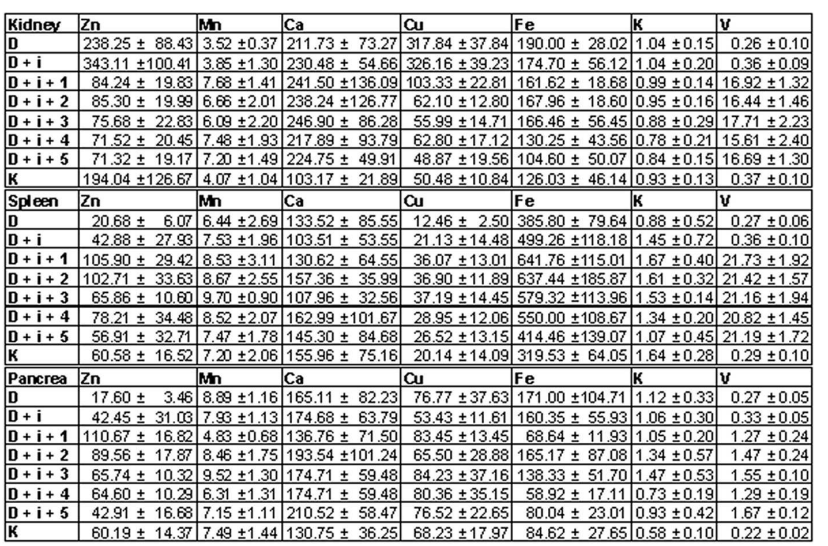

Fig. 9. The summary results of the elemental concentration studies (mean value \pm standard deviation).

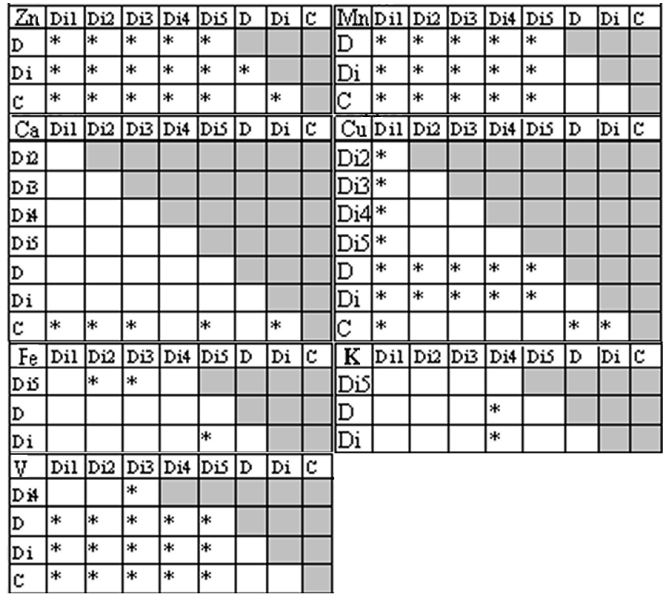

Fig. 10. Significant statistical differences in elemental concentration in kidneys between tested groups of animals (fields marked by ${ }^{*}$ ). See the text below for explanations of field names. Empty rows are omitted.

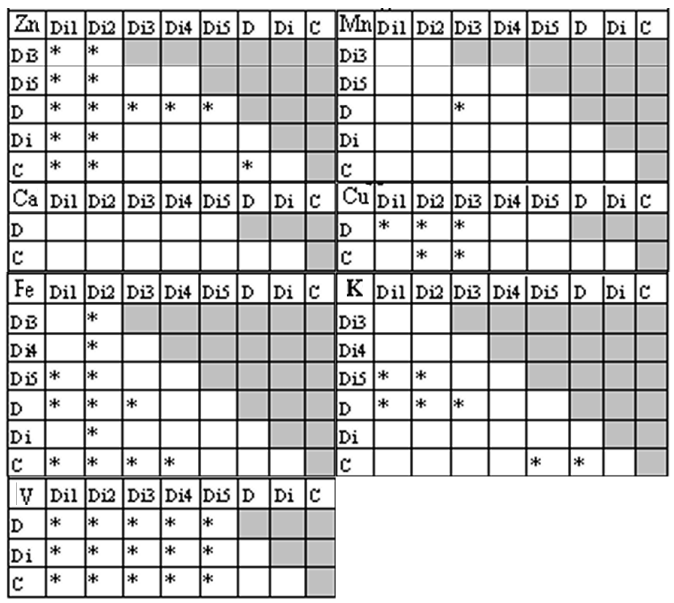

Fig. 11. As in Fig. 10, but for spleens. 


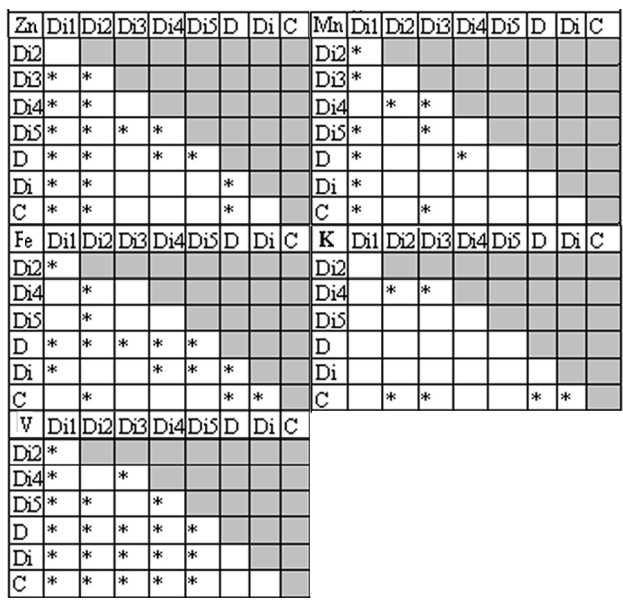

Fig. 12. As in Fig. 10, but for pancreas.

\subsection{Kidneys}

In vanadium treated groups (Di1-Di5) Zn concentration was about twice lower than in diabetic not treated groups $(\mathrm{D}, \mathrm{Di})$ and in the control one $(\mathrm{C})$. Also the insulin injection showed an influence on $\mathrm{Zn}$ concentration, but not as high as vanadium complexes. Insulin solution used in this experiment contains zinc salts and the increase in zinc concentration has been expected. Indeed, the vanadium influence on $\mathrm{Zn}$ concentration in this organ was observed. No correlation was observed between vanadium oxidation state or ligand on this concentration.

There is an influence of supplementation with complex no. 1, 2, 3, and 5 and also of insulin injection on Ca concentration in kidneys, as compared to the control group. The first three complexes were composed of vanadium at $+\mathrm{V}$ oxidation state but differed in ligands, while the 5 th complex contains the vanadium at $+\mathrm{IV}$ oxidation state.

Vanadium supplementation had an influence on $\mathrm{Fe}$ concentration on kidney, but only in 2nd, 3rd complexes and in insulin injection, as compared to the Di5 group. The influence of oxidation state of vanadium was observed in groups 2 and 5 (where the ligands were the same). The oxidation state and dimethyliation in complex 3 increase the Ca concentration in kidney, as compared to the group supplied with the 5th complex. Similar result was observed in the action of insulin injection.

There were also some differences in vanadium concentration in kidneys. Evidently, it was higher in vanadium treated groups as compared to the not treated one. There was also an influence of the vanadium oxidation state and the ligand which was visible in tested groups 3 and 4 .

Vanadium supplementation increases the Mn concentration in kidneys, as compared to the other tested groups.

Vanadium supplementation raises the $\mathrm{Cu}$ concentration in kidneys, as compared to the diabetic not treated groups. In diabetic groups treated with complex 1 , the $\mathrm{Cu}$ concentration is higher, as compared to the other vanadium treated groups and the healthy one, while it is lower as compared to the D and Di group.

The presence of diabetic has an influence on the $\mathrm{Cu}$ concentration in kidneys, as compared to the control healthy group. In these groups the $\mathrm{Cu}$ level is about 6 times higher.

Only in complex no. 4 an influence on potassium concentration in kidneys was observed as compared to the diabetic groups ( $\mathrm{D}$ and $\mathrm{Di}$ ).

Kidney as excretory organ plays an important role influencing the total element concentration also in other tissues. In this moment it is very difficult to discuss about the obtained results, but the collected data provide the basis to consider the total organ element concentration as a very sensitive marker for chronic disease and element supplementation.

\subsection{Spleens}

Vanadium supplementation has an influence on zinc concentration in spleen. In all vanadium treated groups this concentration was higher as compared to the diabetic group, but only supplementation of complexes 1 and 2 had an influence as compared to the Di group, while complexes 1,2 , and 5 had an influence on $\mathrm{Zn}$ concentration as compared to the healthy group. There is a statistical difference in concentration of zinc in the group Di1, Di2 as compared to Di3. All these complexes had the same oxidation state of vanadium but differed in ligands. The statistical difference between group Di1, Di2, and Di5 suggests the influence of vanadium oxidation state on this concentration. The first and the last one differs in oxidation state of vanadium, while the second and the last one differ both in oxidation state and ligand.

In manganese concentration study only an influence of the treatment with complex no. 3 was observed as compared to the diabetic one, which indicates the influence of the dimethyl group. Copper concentration differs in groups 1, 2, and 3 as compared to the diabetic one. The oxidation state of vanadium is the same in all of these complexes. The influence of vanadium supplementation (Di2 and Di3) on $\mathrm{Cu}$ concentration in rats' spleen was also observed as compared to the healthy one. Again, the complexes differed in ligands but were characterized by the same oxidation state.

Iron concentration in spleen was lower in groups of rats treated with vanadium complexes as compared to the healthy group except the complex no. 5. Also the concentration on iron was different in group Di1-Di3 as compared to the diabetic group, where the oxidation state of vanadium was $+\mathrm{V}$. There are statistical differences in iron concentration between groups Di3-Di5, Di, D, and $\mathrm{C}$, as compared to the Di2 group. Also the Di1 and Di5 groups show a statistical difference.

Iron concentration in spleen is the highest among all examined tissues. Naturally, spleen plays a role in conversion of blood red cells and thus a high concentration is expected as compared to other tissues.

Potassium concentration is about twice higher in diabetic groups treated with complexes with vanadium oxidation state $+\mathrm{V}$, as compared to the diabetic not treated 
group. Two of them (Di1 and Di2) show a raised potassium concentration as compared to Di5 group. There is also statistical difference between groups D and Di5 and also D and C, where the level of potassium in the first pair of groups is lower.

In spleen, the increase in vanadium concentration in diabetic vanadium treated groups (Di1-Di5) was observed as compared to the three not treated groups (D, Di, C).

There is no influence of the vanadium supplementation on calcium concentration in spleen in all of the tested groups.

\subsection{Pancreas}

Treatment with complexes no. 1 and 2 increases the zinc concentration in pancreas as compared to all tested groups, while complex no. 5 lowers it as compared to other vanadium treated groups. Also the presence of diabetes decreases the $\mathrm{Zn}$ concentration in pancreas. Zinc is an element which plays an important role in insulin secretion. Destruction of $\beta$-cells by streptozotocin decreases zinc concentration in this tissue. Observation of zinc increase in diabetic vanadium treated rats provides information about possible mechanism of vanadium antidiabetic activity.

Complex 1 has caused the lowest manganese concentration in pancreas as compared to other groups, while complex 4 has increased the concentration of manganese, as compared to groups Di2 and Di3. Complex no. 3 has raised the $\mathrm{Mn}$ concentration in pancreas as compared to group Di5 and C, while complex 4 has increased it as compared to the diabetic group.

Application of all of the tested vanadium complexes has influenced the iron concentration in pancreas as compared to diabetic group, but only complexes 1,4 and 5 have influenced this concentration as compared to the Di group.

The presence of diabetes increases the iron concentration in pancreas by the factor of about 2, as compared to the healthy group, while the insulin injection lowers it. There is a statistical difference between the iron concentration in pancreas supplemented with complex no. 1 and 2 , where the vanadium oxidation state is the same $(+\mathrm{V})$, but the ligands are different. The influence on this concentration depending on the oxidation state of vanadium is reflected in the difference between the group Di2 as compared to Di4 and Di5.

The presence of diabetes raises the potassium concentration in pancreas as compared to the healthy group. This action is similar to the influence of complex no. 2 and 3, where this concentration is higher as compared to the control (C) and Di4 group.

Vanadium supplementation increases the vanadium concentration in pancreas as compared to the not treated groups. There is a statistical difference between group Di1 and Di2, and also between Di4 and Di5, where the influence of vanadium oxidation state and ligand can be observed. This observation is also visible in the difference between group Di2 and Di5. The influence of vanadium oxidation state and the ligand on the vanadium concentration is shown in the difference between Di3 and Di4, while only the influence of ligand is shown in the difference between Di4 and Di5 (both characterized by the $+\mathrm{V}$ vanadium oxidation state). There is no influence of vanadium supplementation on calcium and copper concentration in pancreas.

\section{Conclusions}

The PIXE method is a sensitive tool which can detect concentration of trace elements in the level of about $1 \mu \mathrm{g}$ in $1 \mathrm{~kg}$ of a sample (as shown e.g. in Fig. 7). It is a non-destructive, multi-elemental method, so it may be used as a complementary technique with other analytical methods. As shown in this research, the method can be very useful in application to biological samples.

The presented results illustrate the change in microand macro-elements concentration in living organisms in disease (streptozotocin induced diabetes type I) and elemental supplementation (vanadium treatment). This observation can be helpful in better understanding of the mechanisms of diabetes and other diseases evaluation. Also the proper design of supplementation elemental content can be considered as the possible way to reduce the diabetic complications. In future, this knowledge may benefit in better disease treatment. It is expected that in other diseases similar mechanisms can be observed and they can be useful in future in disease treatment.

\section{References}

[1] ADA, Diab. Care 20, 43 (1997).

[2] L. Stryer, Biochemistry, PWN, Warszawa 2001 (in Polish).

[3] G.B. Dubyak, A. Kleinzeller, J. Biol. Chem. 255, 5306 (1980).

[4] A.M. Kordowiak, R. Trzos, Post. Biol. Kom. 21, 293 (1994).

[5] J.H. McNeill, J. Wang, V.G. Yuen, Metabolism 50, 667 (2001).

[6] C. Djordjevic, G.L. Wampler, J. Inorg. Biochem. 25, 51 (1980).

[7] M. Blusz, M.Sc. Thesis, Medical College Jagiellonian University, Kraków 2005.

[8] V. Valkovic, IAEA-TECDOC-300, IAEA, Vienna 1983.

[9] A.Z. Hrynkiewicz, E. Rokita, Research methods of physics in biology, medicine and environmental protection, PWN, Warszawa 1999 (in Polish).

[10] M. Govil, Current Sci. 80, 1542 (2001).

[11] R.M. Parr, IAEA-TECDOC-197, IAEA, Vienna 1980.

[12] The GupixWin Manual and User-Guide, University of Guelph, Guelph 2005.

[13] J. Greń, Mathematical statistics, Programmers handbook, PWN, Warszawa 1984 (in Polish). 